\title{
Primer registro de Tlacuatzin canescens, (Mammalia, Didelphimorphia: Marmosidae) en Veracruz, México
}

\author{
Alvar González Christen ${ }^{1}$ y Nallely Verónica Rodríguez Santiago ${ }^{1}$
}

Introduction: The Mexican Mouse Opossum (Marmosa mexicana) and the grayish mouse opossum (Tlacuatzin canescens) were the only two known species of tribu Marmosini (Mammalia, Didelphimorphi, Didelphidae) in México. The genus Tlacuatzin and its species Tlacuatzin canescens are endemic. Its distribution extends from the south of the State of Sonora to the State of Chiapas. It is also found on the Islas Marias, in the state of Jalisco, in the Yucatán Peninsula, and in the State of Puebla. So far, T. canescens has not been previously sighted in the State of Veracruz or in the north coast of the Gulf of Mexico. This report describes the first official sighting of $T$. canescens from Veracruz, México.

Methods: The records were obtained: a) from voucher specimens deposited at the Colección Mastozoológica, Instituto de Investigaciones Biológicas, Universidad Veracruzana, and b) by accidentally trapping these mammals in botanical surveys conducted at the El Centro de Investigaciones Costeras La Mancha, Instituto de Ecología A.C. and its surroundings.

Results: The presence of $T$. canescens in Veracruz State was documented by nine records collected at three different localities, from the central region of Veracruz, México. All the locations were found in deciduous forest areas.

Discussion and Conclusions: These new records increase the species' known geographic distribution in 139 $\mathrm{km}$, on a straight line from the nearest locality found in the state of Puebla. It is important to recall that these locations are all located on both sides of the Sierra Madre Oriental Mountain system (average altitude higher than 2000 meters above sea level, between localities), which serves as a barrier between the populations of Puebla and Veracruz.

Key words: Didelphimorphia, Geographic distribution, Local distribution, Marsupial, Opossum-souris cendré

El ratón tlacuache mexicano (Marmosa mexicana) y el ratón tlacuache grisáceo (Tlacuatzin canescens) son las dos únicas especies de la tribu Marmosini (Mammalia, Didelphimorphia, Didelphidae) en México. El género Tlacuatzin y la especie Tlacuatzin canescens son endémicos de México, su distribución abarca desde el sur del estado de Sonora hasta el estado de Chiapas, también se localiza en las Islas Marías, en la península de Yucatán y en el Estado de Puebla. Su presencia en el estado de Veracruz se confirma con los nueve registros aquí reportados, provenientes de localidades ubicadas en la porción central del estado. Los registros se obtuvieron mediante: a) revisión de ejemplares depositados en colección y b) captura y liberación. Las localidades presentan vegetación de selva baja caducifolia. Estos nuevos registros incrementan la distribución geográfica conocida de la especie en $139 \mathrm{~km}$

'Instituto de Investigaciones Biológicas Universidad Veracruzana. Av. Luis Castelazo S/n Col. Industrial Animas, Xalapa 91190, Veracruz, México. Email: agonzalez@uv.mx (AGC), nayver82@gmail.com (NVRS).

*Corresponding author 
en línea recta desde la localidad más cercana en el Estado de Puebla. Es importante resaltar que estas localidades se encuentran a ambos lados de la Sierra Madre Oriental (altitud superior a 2,500 metros sobre el nivel del mar), que impide actualmente el intercambio entre las poblaciones de Puebla y Veracruz.

Palabras Clave: Didelphimorphia, distribución geográfica, distribución local, ratón tlacuache.

En México la tribu Marmosini de la familia Didelphidae en el Orden Didelphimorphia, está representada por dos géneros, cada uno con una especie coloquialmente conocidas como: ratón tlacuache gris, tlacuachin o tlacuatzin Tlacuatzin canescens (J. A. Allen 1893) y ratón tlacuache mexicano Marmosa mexicana Merriam, 1897 (Gardner 1993; Ramírez-Pulido et al. 2014). La primera es una especie endémica de México, politípica con tres subespecies: T. canescens canescens; T. c. insularis y T. c. gaumeri (Ramírez-Pulido et al. 2014), esta especie se presenta principalmente en las tierras bajas tropicales de la vertiente occidental de México desde Sinaloa hasta Chiapas, penetrando hacia el centro del país en Puebla a través de la cuenca del rio Balsas. Además, existen poblaciones aisladas en las Islas Tres Marías en Nayarit y en Campeche y Yucatán (Zarza et al. 2003; Brown 2004; Vargas et al. 2004; Ceballos 2005; Hernández-Cardona. et al. 2007; Ramírez Pulido et al. 2007; Retana et al. 2010; Figura 1). La especie T. canescens, no ha sido previamente registrada en el estado de Veracruz, por lo que el objetivo de este artículo es documentar su presencia en la entidad, lo que amplía la distribución conocida de T. canescens en la República Mexicana.

Introducción

\section{Material y Métodos}

La presencia de T. canescens en Veracruz se confirmó mediante: a) revisión de ejemplares depositados en la Colección Mastozoológica del Instituto de Investigaciones Biológicas de la Universidad Veracruzana (Registro de Colección Científica, Clave: VER.- MAM-191-10-06 SEMARNAT) y b) captura y liberación. Los ejemplares depositados en la colección provienen de las localidades Plan del Rio, Municipio de Emiliano Zapata y Playa Norte Municipio de Veracruz, el resto de los ejemplares se registraron en el Centro de Investigaciones Costeras La Mancha, Instituto de Ecología A. C. (CICOLMA) y sus alrededores en el municipio de Actopan (Tabla 1; Figura 2).

\begin{tabular}{lccclc}
\hline Id & LN & LW & Altitud (msnm) & Localidad & Tipo de registro \\
\hline 1 & $19^{\circ} 35^{\prime} 53.0^{\prime \prime}$ & $96^{\circ} 22^{\prime} 45.8^{\prime \prime}$ & 9 & CICOLMA & $\mathrm{C} / \mathrm{L}$ \\
2 & $19^{\circ} 36^{\prime} 2.3^{\prime \prime}$ & $96^{\circ} 22^{\prime} 46.6^{\prime \prime}$ & 29 & CICOLMA & $\mathrm{C} / \mathrm{L}$ \\
3 & $19^{\circ} 36^{\prime} 4.7^{\prime \prime}$ & $96^{\circ} 22^{\prime} 46.4^{\prime \prime}$ & 34 & CICOLMA & $\mathrm{C} / \mathrm{L}$ \\
4 & $19^{\circ} 36^{\prime} 3.79^{\prime \prime}$ & $96^{\circ} 22^{\prime} 49.7^{\prime \prime}$ & 33 & CICOLMA & $\mathrm{C} / \mathrm{L}$ \\
5 & $19^{\circ} 37^{\prime} 1.41^{\prime \prime}$ & $96^{\circ} 23^{\prime} 4.84^{\prime \prime}$ & 95 & Eco-Guías & $\mathrm{C} / \mathrm{L}$ \\
6 & $19^{\circ} 36^{\prime} 51.8^{\prime \prime}$ & $96^{\circ} 23^{\prime} 4.51^{\prime \prime}$ & 72 & Eco-Guías & $\mathrm{C} / \mathrm{L}$ \\
7 & $19^{\circ} 36^{\prime} 49.5^{\prime \prime}$ & $96^{\circ} 23^{\prime} 4.76^{\prime \prime}$ & 73 & Eco-Guías & $\mathrm{C} / \mathrm{L}$ \\
3225 & $19^{\circ} 23^{\prime} 46.8^{\prime \prime}$ & $96^{\circ} 37^{\prime} 32.1^{\prime \prime}$ & 330 & Plan del Río & $\mathrm{P} / \mathrm{C}$ \\
3607 & $19^{\circ} 13^{\prime} 20.0^{\prime \prime}$ & $96^{\circ} 10^{\prime} 22.8^{\prime \prime}$ & 8 & Playa Norte & $\mathrm{P} / \mathrm{C}$ \\
\hline
\end{tabular}

Tabla 1. Puntos de colecta y avistamiento de Tlacuatzin canescens en el centro de Veracruz. Registro (naturaleza del ejemplar): $\mathrm{C} / \mathrm{L}=$ Captura liberación; P/C Piel y Cráneo. 


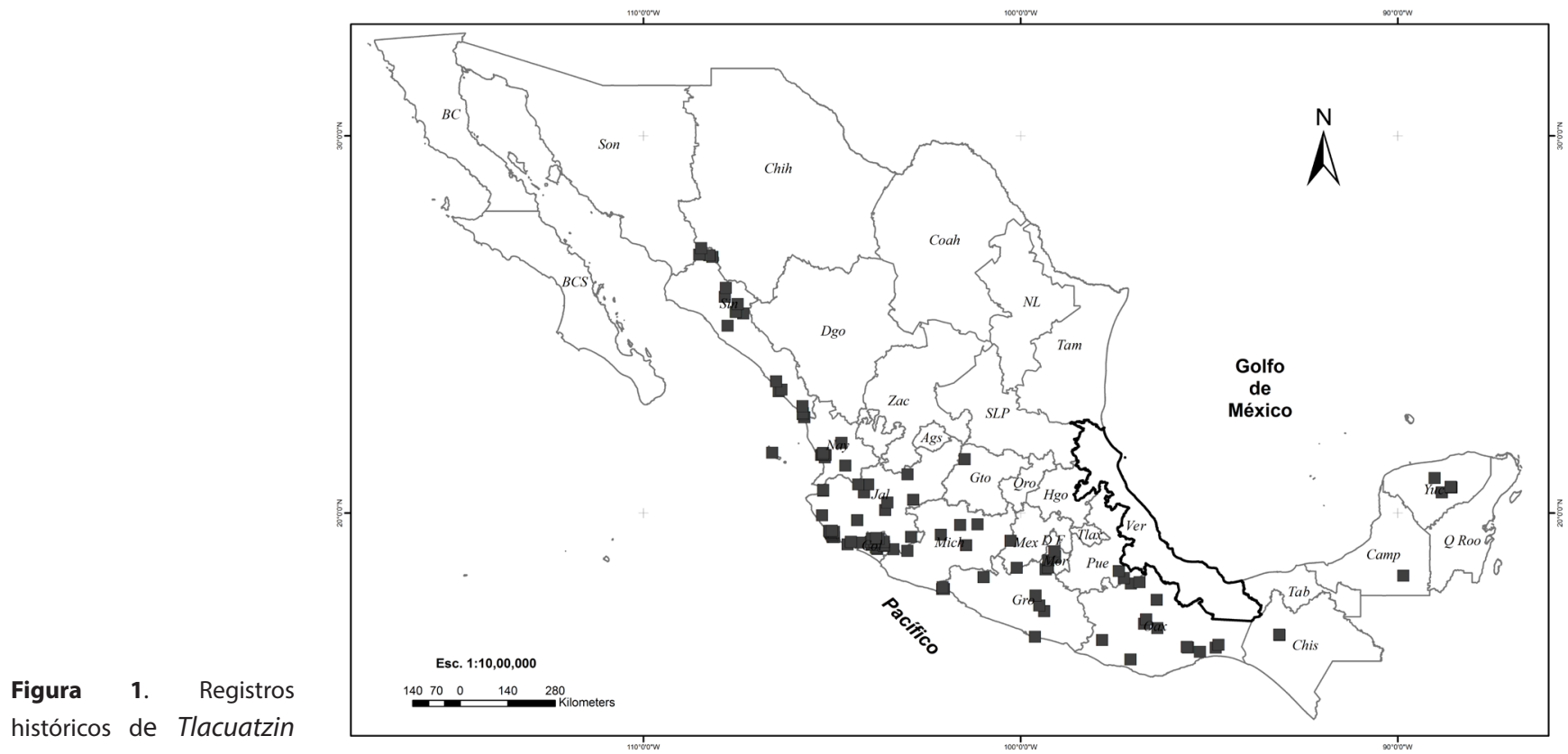

canescens en México.

El tipo de vegetación presente en las localidades de colecta corresponde a la selva baja caducifolia, esta vegetación se distribuye en México a partir de la vertiente del Pacífico y hacia la Península de Yucatán, contando con algunos macizos boscosos aislados en el Golfo de México en los estados de Tamaulipas, Veracruz y Yucatán. En Veracruz, esta vegetación se presenta principalmente en la porción central del estado, en altitudes menores a los 850 metros sobre el nivel del mar, donde se presentan climas semicálido húmedo $((A) C(m))$ y cálido subhúmedo $\left(A w_{1}\right.$ y $\left.A w_{2}\right)$, con un intervalo de temperatura anual entre 21 a $28^{\circ} \mathrm{C}$ y con una precipitación anual de 300 a 1,800 mm anuales. En el estado, son especies vegetales típicas de esta asociación: chicozapote (Manilkara zapota), palo mulato (Bursera simaruba), rama tinaja (Trichilia havanensis), zapote prieto (Diospyros digyna), barbasco (Dioscorea mexicana) y papaya cimarrona (Jacaratia dolichaula). En la Tabla 1 se muestran las coordenadas de los sitios de colecta y registro de los T. canescens reportados del centro de Veracruz.

Los ejemplares incorporados a la colección fueron capturados en estudios prospectivos de fauna mediante el uso de 40 trampas tipo Sherman colocadas en el piso cada diez pasos y cebadas con una mezcla de hojuelas de avena, vainillina y agua, para su incorporación museográfica se prepararon en piel y cráneo. Los ejemplares reportados de CICOLMA se capturaron accidentalmente al caer dentro de trampas para hojarasca, se les tomo fotografías y liberó en el sitio.

Para la determinación taxonómica del material depositado de la colección, a) se analizaron pelos de guardia conservados siguiendo a Juárez et al. (2010) y b) fenotípicamente con el apoyo de claves dicotómicas (Hall 1981) y descripciones monográficas (Alonso y Medellín 1992; Zarza et al. 2003). Para corroborar la correcta identificación, se revisaron y compararon ejemplares de la especie depositados en las colecciones mastozoológicas: Colección Nacional de Mamíferos del Instituto de Biología (CNMA); Colección del Museo de Zoología de la Facultad de Ciencias (MZFC-M), ambas de la Universidad Nacional Autónoma de México y la colección de mamíferos de la Universidad Autónoma Metropolitana Iztapalapa (UAMI). Paralelamente, con el fin de confirmar la probable ocurrencia de otros ejemplares se realizó una búsqueda documental electrónica utilizando los portales Global Biodiversity 
Información Facility (GBIF), ManisNet, UNIBIO y CONABIO (Consultados el 12/05/2014); simultáneamente fueron consultados otros documentos que proporcionaron información acerca de su distribución geográfica y otros registros (Alonso y Medellín 1992; Miranda 1997; Ramírez-Pulido 1997; Velázquez y Romero 1998; López-Wilchis 2003; Muñoz-Alonso y March 2003; Sánchez-Cordero y Linaje 2003; Zarza et al. 2003; Gómez-Escamilla 2004; CervantesReza 2005; Briones-Salas 2006; Chávez-León 2006; López-Vidal 2006; Ramírez-Pulido y Martínez-Vázquez 2007; López-Vidal et al. 2008; González-Christen 2011). La identificación de los ejemplares capturados en el CICOLMA y sus alrededores, se realizó en el sitio, basados en los caracteres fenotípicos externos, su identificación se corroboró posteriormente en las instalaciones de la Colección a través de la revisión de las fotografías tomadas en sitio.

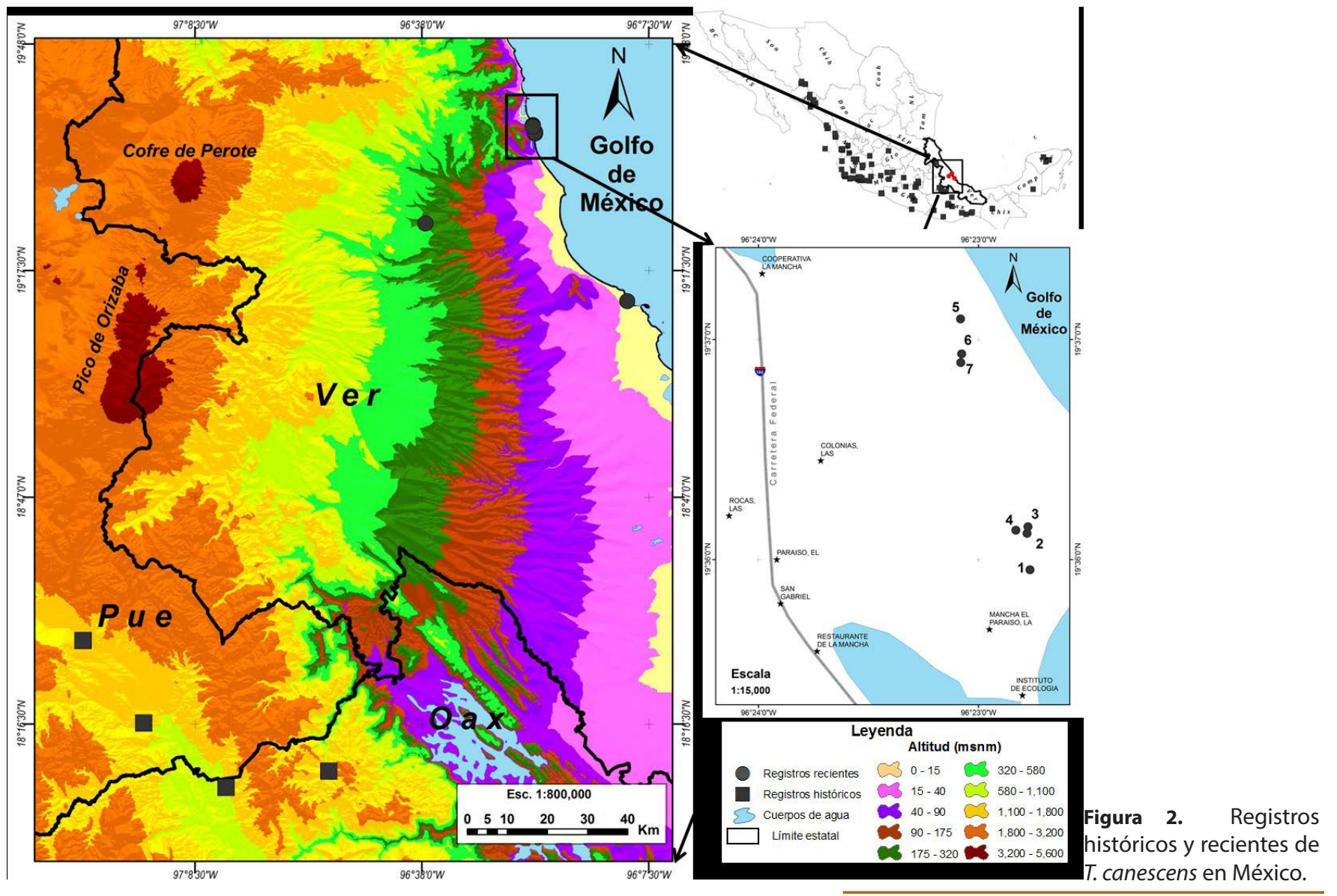

Las coordenadas geográficas de los sitios de registro, así como las obtenidas de la búsqueda documental fueron colocados en un espacio-mapa con la ayuda de un sistema de información geográfica (Arc Map 10.1), para medir la distancia entre los registros históricos más cercanos al estado de Veracruz con los registros obtenidos durante las colectas para esta contribución.

Resultados

Fenotípicamente, Marmosa mexicana y Tlacuatzin canescens son muy parecidas, aunque se pueden distinguir entre sí por el pelaje, ya que T. canescens presenta un pelaje más corto y de color gris, bicolor, pelos con médula escalonada uniserial y escama ondulada irregular (Monroy y Rubio 2003; Baca y Sánchez 2004), mientras que en M. mexicana presenta un pelaje más largo y de color rojizo, bicolor, pelos con médula escalonada uniserial y las escamas tipo 
en forma de diamante (Baca y Sánchez 2004). En T. canescens el antifaz formado por el anillo ocular es grande, de coloración negro y se proyecta sobre los frontales hacia la parte alta de la cabeza, en tanto que en M. mexicana no alcanza la parte superior de la cabeza. En el cráneo T. canescens desarrolla unos prominentes bordes supraorbitales en forma de ala, el paladar presenta fenestras palatales y a la altura del segundo molar superior presenta además otras fenestras palatales accesorias. En M. mexicana solo se desarrollan unos bordes supraorbitales de manera incipiente y únicamente presenta las fenestras palatales normales (Figura 3). El proceso rostral del premaxilar de M. mexicana es más prominente que en T. canescens (Villa y Cervantes 2002; Baca 2004, Ceballos 2005; Medellín 2005; Rossi et al. 2010).

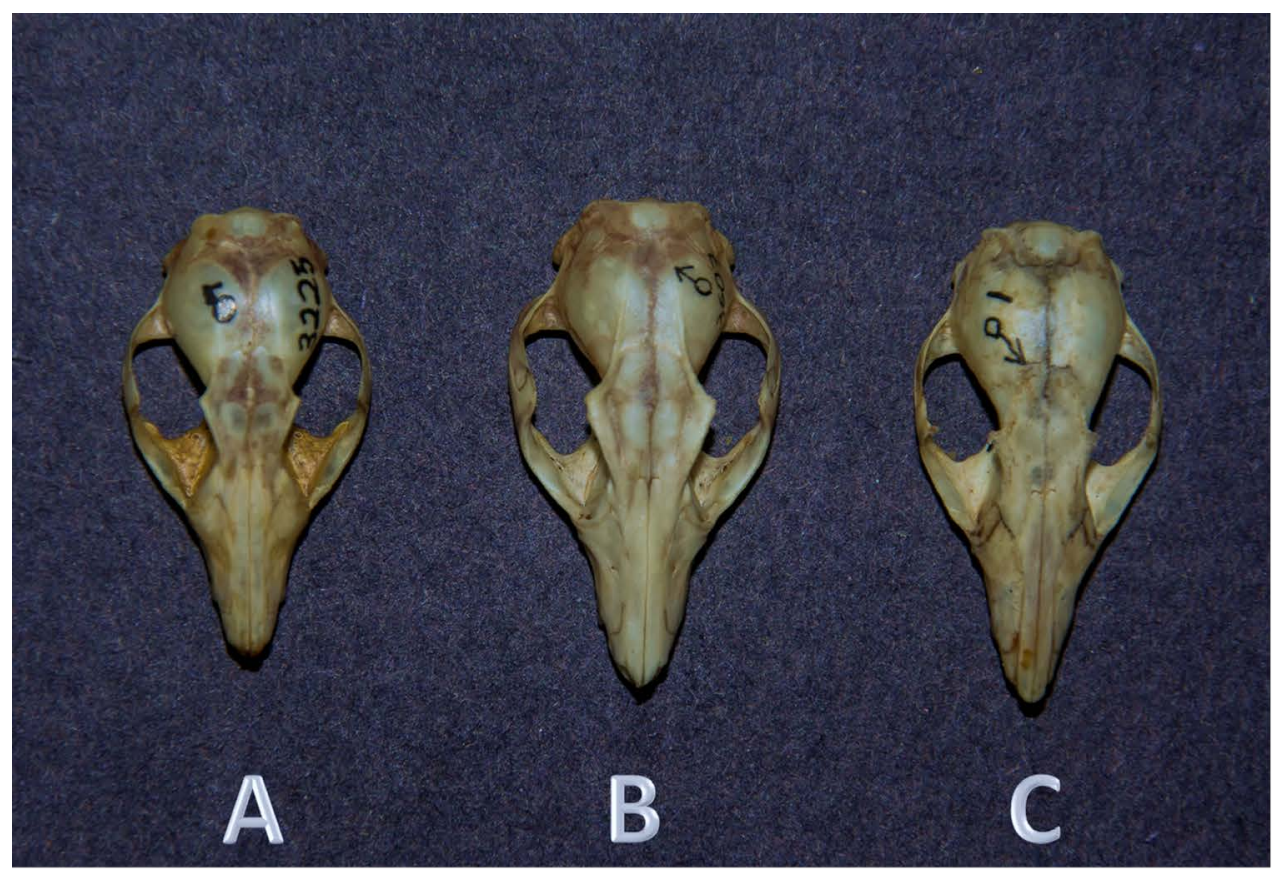

Figura 3. Vista dorsal y ventral del Cráneo de Marmosa mexicana (A) y Tlacuatzin canescens (B y C).

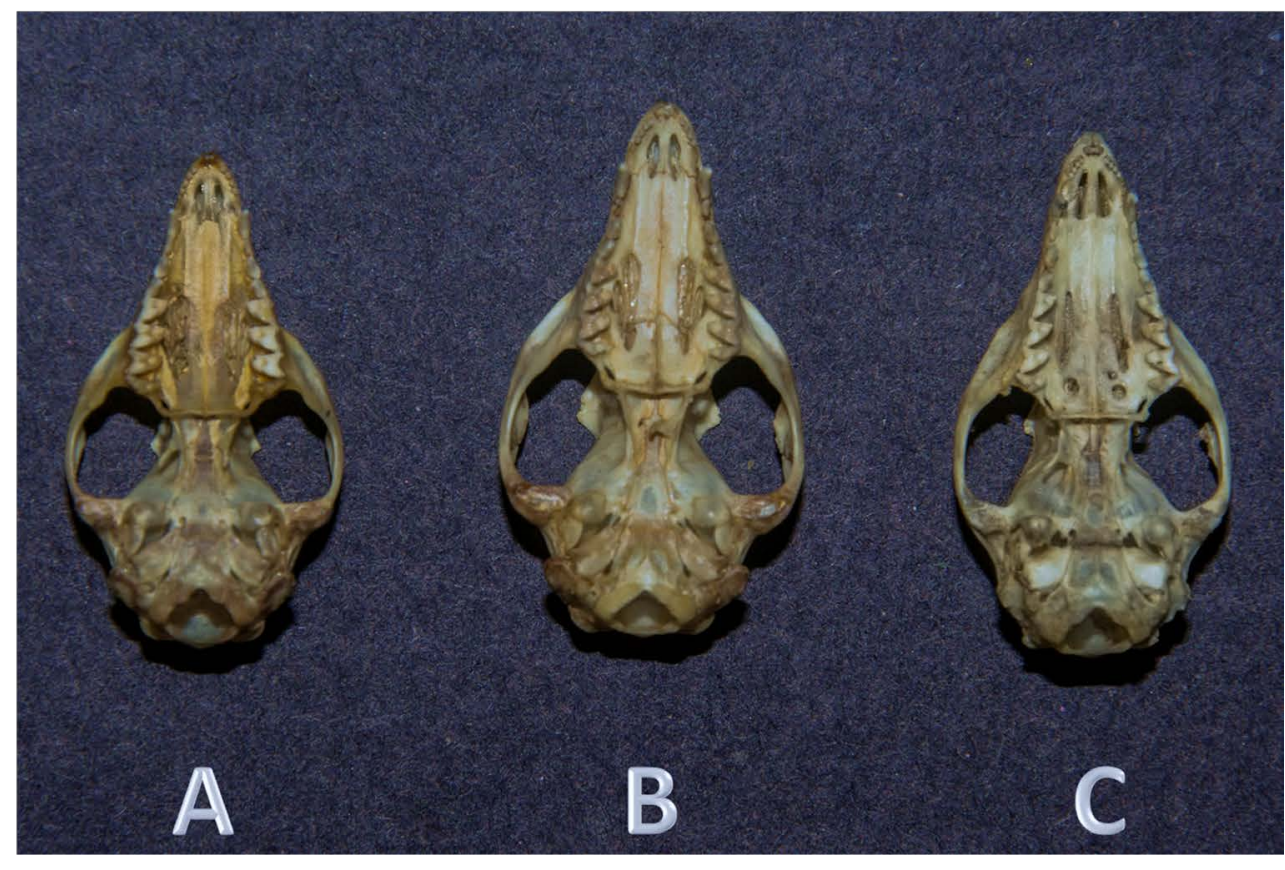


Los reportes de $T$. canescens obtenidos mediante la búsqueda documental ascienden a 483, aunque solo 280 cuentan con las coordenadas de las localidades de colecta (Figura 1); a ellos se suman los registros obtenidos en Veracruz, haciendo un total de 289 registros con datos geográficos. Documentamos nueve registros nuevos de T. canescens, de los cuales, sólo dos ejemplares fueron colectados e incorporados a la Colección de Mamíferos bajo el resguardo del Instituto de Investigaciones Biológicas de la Universidad Veracruzana (CIB), el resto de los siete individuos fueron liberados en los sitios donde fueron capturados (Tabla 1).

\begin{tabular}{cccccccccccccccc}
\hline \multirow{2}{*}{ CAT } & SEX & ALMA & LOMA & LAFM & HMXD & ALRO & LOCR & LONA & LOFR & ANCI & ANIN & ANCC & LOCO & LOFI \\
\hline 3225 & $M$ & 6.96 & 20.90 & 4.38 & 12.00 & 2.77 & 31.00 & 12.80 & 8.85 & 15.90 & 4.52 & 11.90 & 30.90 & 2.71 \\
3607 & $M$ & 8.62 & 25.00 & 4.07 & 9.45 & 2.93 & 33.70 & 14.60 & 10.00 & 17.60 & 5.20 & 12.80 & 33.50 & 2.84 \\
\hline
\end{tabular}

Los acrónimos significan las medidas craneales, ALMA (Altura de la mandíbula), LOMA (longitud de la mandíbula), LAFM (Largo de la base del I1 sobre la base del dentario al foramen del mentón), HMXD (longitud de la hilera maxilar de dientes), ALRO (altura del rostro), LOCR (longitud total del cráneo), LONA (longitud de los nasales), LOFR (longitud de los frontales), ANCI (anchura del arco cigomático), ANIN (anchura interorbital), ANCC (anchura de la caja craneal), LOCO (longitud cóndilobasal), LOFI (longitud del foramen incisivo).

El primer espécimen de $T$. canescens para el estado de Veracruz se recolecto el 8 de Noviembre del año 2006 en la localidad de Plan del Río (CIB 3225) y correspondió a un individuo juvenil con las siguientes medidas convencionales: longitud total $232 \mathrm{~mm}$, longitud cola $121 \mathrm{~mm}$, longitud pata $19 \mathrm{~mm}$, longitud oreja $22 \mathrm{~mm}$. El segundo espécimen registrado fue un macho adulto, el 21 de Junio del año 2008 en Playa Norte, Punta Gorda (CIB 3607) y presenta las siguientes medidas convencionales: 266, 143, 20, 24. Las medidas craneales de ambos ejemplares se muestran en el Tabla 2. En los alrededores del Centro de Investigaciones Costeras La Mancha del Instituto de Ecología A. C. (CICOLMA), en 2013 se confirmaron otros siete registros que corresponden a T. canescens.

Discusión

La importancia de estos registros radica en que se documenta por primera vez la presencia del género Tlacuatzin y de la especie T. canescens en el estado de Veracruz y también en el Golfo de México, por tanto también es una ampliación de distribución de más 130 km en línea recta. Lo consideramos registro notorio puesto que no se conocían (género) en el estado, ni en la costa del Golfo de México, además se reporta la presencia en tres diferentes localidades de colecta (nueve individuos total registrados en nueve diferentes puntos de colecta) con una considerable distancia entre tres de los sitios referidos $(\approx 40-60 \mathrm{~km}$ entre ellos). Lo que nos confirma la presencia del género y la especie en esa región del estado, no es un registro accidental o que se debiera confirmar por su rareza. Esto tiene implicaciones de carácter biogeográfico que deben ser estudiadas. Estos registros amplían su distribución en el país en 132 km hacia el Este en línea recta respecto a la localidad más cercana, ubicada en la Reserva de la Biosfera Tehuacán-Cuicatlán (RBTC) con fecha de 18/10/1944 hecho por H. O. Wagner (UMMZ-88634), la especie también fue reportada recientemente en la misma RBTC por Ramírez (2007). Con estos hallazgos se incrementa la riqueza de géneros y especies de la mastofauna conocida de Veracruz de 116 géneros y 192 especies a 117 géneros con 193 especies (González-Christen 2012). 
Es importante resaltar que la RBTC que es localidad más cercana se encuentra en la vertiente occidental de la Sierra Madre Oriental, en tanto que los registros veracruzanos corresponden a la Planicie Costera Veracruzana localizada en la vertiente oriental. En esta región la Sierra Madre Oriental alcanza alturas superiores a los 2,500 msnm y tiene un ancho de 50 kilómetros aproximadamente (con diversos tipos climáticos y de vegetación) y constituye una barrera para la dispersión de la especie. Hacia el sur del país en el estado de Campeche aproximadamente a $700 \mathrm{~km}$, se ha reportado la presencia del género Tlacuatzin a 37 km al norte de la región de Calakmul (Vargas-Contreras et al. 2004).

La selva baja caducifolia del Golfo de México estuvo integrada biogeográficamente con la selva baja caducifolia de la vertiente del Pacífico de México, durante el Pleistoceno. La extensión de la selva baja caducifolia del resto del país, probablemente abarco una mayor superficie y a consecuencia de la alternancia de los climas, en la costa del Golfo de México se redujo a los tres grandes fragmentos que se reconocen en la actualidad, convirtiéndose cada uno en un refugio aislado (Challenger 1998). La presencia de estos nuevos registros contribuye a llenar vacíos de información y amplían del área de distribución potencial de esta especie propuesta por Sánchez Cordero y Linaje (2003), así como el área disponible para el género según Sánchez-Cordero et al. (2005).

Los nuevos registros se ubican en la provincia Golfo de México dentro del componente mesoamericano (Morrone y Márquez 2003). En Veracruz de esta provincia se conocían 117 especies de mamíferos, tres especies de carácter endémico (González 2011). La presencia de T. canescens que se reporta aquí y su asociación con los remanentes de selva baja caducifolia en la zona central del estado de Veracruz, resaltan por un lado, la importancia biológica de esta vegetación para los mamíferos y por el otro la necesidad de continuar con los estudios del registro de la mastofauna en esta región de Veracruz.

\section{Agradecimientos}

Agradecemos a L. León Paniagua de la Colección Alfonso Herrera de la Facultad de Ciencias, a F. Cervantes Reza de la Colección Nacional de Mamíferos del Instituto de Biología de la Universidad Nacional Autónoma de México, y a J. Ramírez Pulido de la Colección de Mamíferos de Universidad Autónoma Metropolitana Iztapalapa, por permitirnos la revisión del material depositado en las colecciones a su cargo y por todas las facilidades otorgadas. E. Priego Hernández quien proporcionó las imágenes que corroboran la presencia del Tlacuatzin en los alrededores del CICOLMA, a C. A Delfín Alfonso por su apoyo en la elaboración de los mapas de distribución así como sus comentarios y sugerencias al manuscrito, asimismo a A. González Zamora por sus comentarios y sugerencias al manuscrito, a R. Reyna y los dos revisores anónimos por las observaciones y valiosos comentarios que ayudaron en gran medida a mejorar la calidad de este trabajo.

\section{Literatura citada}

Alonso-Mejía, A., y R. Medellín. 1992. Marmosa mexicana. The American Society of Mammalogists. Mammalian Species 421:1-4.

Baca, I., Y V. Sánchez. 2004. Catálogo de pelos de guardia dorsal en mamíferos del estado de Oaxaca, México. Universidad Nacional Autónoma de México, Serie Zoología 75:383437. 
Briones Salas, M. A. 2006. Incorporación de nuevos registros de mamíferos de la región Sierra Norte de Oaxaca. Instituto Politécnico Nacional. Centro Interdisciplinario de Investigación para el Desarrollo Integral Regional-Oaxaca. Bases de datos SNIB2010CONABIO proyectos No. BA009 y R104. Ciudad de México, México.

Brown, E. B. 2004. Atlas of New World Marsupials Zoology Fieldiania New Series No 102 Field Museum of Natural History.

Ceballos, G. 2005. Tlacuatzin canescens (J. A. Allen, 1893) Tlacuachín. Pp. 100-101 en Los mamíferos silvestres de México (Ceballos, G., y G. Oliva 2005, eds.). Fondo de Cultura Económica, Comisión Nacional para el Conocimiento y Uso de la Biodiversidad. . Ciudad de México, México.

Cervantes Reza, F. A. 2005. Computarización de la Colección Nacional de mamíferos del Instituto de Biología, UNAM. Universidad Nacional Autónoma de México. Instituto de Biología. Bases de datos SNIB2010-CONABIO proyecto No. V043. Ciudad de México, México.

Challenger, A. 1998. Utilización y conservación de los ecosistemas terrestres de México: pasado, presente y futuro. Comisión Nacional para el Conocimiento y Uso de la Biodiversidad, Instituto de Ecología Universidad Nacional Autónoma de México y Sierra Madre. Ciudad de México, México.

Chávez León, G. 2006. Inventario florístico y faunístico del Parque Nacional Barranca del Cupatitzio, Michoacán. Secretaría de Agricultura, Ganadería, Desarrollo Rural, Pesca y Alimentación. Instituto Nacional de Investigaciones Forestales Agrícolas y Pecuarias. Bases de datos SNIB2010-CONABIO proyecto No. AS014. . Ciudad de México, México.

Gardner, A. L. 1993. Order Didelphimorphia Pp. 15-23 en Mammal Species of the World. A Taxonomic and Geographic Reference (Wilson, D. E., y D. M. Reeder, eds.). Smithsonian Institution Press, Washington, EE.UU.

Gómez-Escamilla, M. 2004. Anfibios, reptiles y mamíferos del corredor biológico del norte de Yucatán depositados en las colecciones de la Escuela Nacional de Ciencias Biológicas. Instituto Politécnico Nacional. Escuela Nacional de Ciencias Biológicas. Bases de datos SNIB2010-CONABIO proyecto No. Y013. Ciudad de México, México.

González-Christen, A. 2011. Mamíferos: Distribución, endemismo y estado de conservación. Pp. 579-592 en La biodiversidad en Veracruz: Estudio de Estado (Cruz Angón A., ed.). Comisión Nacional para el Conocimiento y Uso de la Biodiversidad, Gobierno del Estado de Veracruz, Universidad Veracruzana, Instituto de Ecología, A. C. Xalapa, México.

HaLl, E. R. 1981. The mammals of North America. John Wiley and Sons. New York, EE.UU.

Hernández-Cardona, A., L. Lago-Torres, R. Ibarra-González, J. Faller- Menéndez, y Y. PereyraArellano. 2007. Registro del Tlacuachin (Tlacuatzin canescens) en el área de conservación El Zapotal, en el noroeste del estado de Yucatán. Revista Mexicana de Mastozoología 11:85-90.

Juárez, D., Ch. Estrada, M. Bustamante, Y. Quintana, J. Moreira, y J. López. 2010. Guía ilustrada de pelos para identificación de mamíferos mayores y medianos de Guatemala. Universidad de San Carlos Guatemala. Guatemala, Guatemala.

López VIDAL, J. C. 2006. Computarización de las colecciones de vertebrados terrestres de la Escuela Nacional de Ciencias Biológicas, IPN Fase 1: Estado de México, Hidalgo, San Luis Potosí y Tlaxcala. Instituto Politécnico Nacional. Escuela Nacional de Ciencias Biológicas. Bases de datos SNIB2010-CONABIO. Proyecto No. BC004. Ciudad de México, México. 
López-Vidal, J. C., C. Elizalde-Arellano, J. I. Campos-Rodríguez, N. Matías-Ferrer, N. González, y S. Murillo-Jiménez. 2008. Computarización de las colecciones de vertebrados terrestres de la Escuela Nacional de Ciencias Biológicas, IPN - Fases 2 y 3. Instituto Politécnico Nacional. Escuela Nacional de Ciencias Biológicas. Bases de datos SNIB2010-CONABIO. Fase 2. Proyecto No. CC002. Ciudad de México, México.

López-Wilchis, R. 2003. Base de datos de Los mamíferos de México depositados en colecciones de Estados Unidos y Canadá. Universidad Autónoma MetropolitanaIztapalapa. http://investigacion.izt.uam.mx/mamiferos/ 07/08/2013

Medellín, R. 2005. Marmosa mexicana Merriam, 1897 Ratón Tlacuache. Los mamíferos silvestres de México (Ceballos, G., y G. Oliva, eds.). Fondo de Cultura Económica, Comisión Nacional para el Conocimiento y Uso de la Biodiversidad. . Ciudad de México, México.

Miranda, A. 1997. Deforestación y fragmentación del hábitat: consecuencias ecológicas sobre la fauna de mamíferos de la selva tropical estacional. Universidad Nacional Autónoma de México. Instituto de Ecología. Bases de datos SNIB2010-CONABIO proyecto No. B033. Ciudad de México, México.

Morrone J. J., y J. Márquez. 2003. Aproximación a un Atlas Biogeográfico de México: Componentes bióticos principales y provincias biogeográficas. Pp 217-220 en Una perspectiva latinoamericana de la Biogeografía (Morrone, J. J., y J. Llorente-Bousquets, eds.). Facultad de Ciencias, Universidad Nacional Autónoma de México. Ciudad de México, México.

Muñoz Alonso, L. A., y I. J. March Mifsut. 2003. Actualización y enriquecimiento de las bases de datos del proyecto de evaluación y análisis geográfico de la diversidad faunística de Chiapas. El Colegio de la Frontera Sur. Bases de datos SNIB2010-CONABIO proyectos No. U014 y P132. Ciudad de México, México.

Ramírez Pulido, J. 1997. Análisis morfofenético de las poblaciones alopátricas de Thomomys umbrinus (Rodentia: Geomyidae) en la provincia volcánico-transversa. Universidad Autónoma Metropolitana-Unidad Iztapalapa Bases de datos SNIB2010-CONABIO proyecto No. B011. Ciudad de México, México.

Ramrez-Pulido, J., y J. Martínez-Vázquez. 2007. Diversidad de los mamíferos de la Reserva de la biósfera Tehuacán-Cuicatlán, Puebla-Oaxaca, México. Universidad Autónoma Metropolitana-Unidad Iztapalapa. Bases de datos SNIB2010-CONABIO proyecto No. BK022. Ciudad de México, México.

Ramírez-Pulido J., N. González-Ruiz, A. L. Gardner, y J. Arroyo-Cabrales. 2014 List of Recent Land Mammals of Mexico, 2014. Special Publications of the Museum of Texas Tech University 63:1-69

Retana, G. O., M. Weber, y D. Guzmán. 2010. Mamíferos terrestres en Campeche. Pp xx en La Biodiversidad en Campeche: Estudio de Estado (Villalobos-Zapata, G. J., y J. Mendoza Vega, coord.). Comisión Nacional para el Conocimiento y Uso de la Biodiversidad (CONABIO), Gobierno del Estado de Campeche, Universidad Autónoma de Campeche, El Colegio de la Frontera Sur. México. Campeche, México.

Rossi, R. A., Robert S. Voss, y Darrin P. Lunde. 2010. A revision of the didelphid marsupial genus Marmosa part 1. The species in Tate's 'mexicana' and 'mitis' sections and other closely related forms Bulletin of The American Museum of Natural History Number 334:1-83. 
Sánchez-Cordero, V., y M. A. Linaje. 2003. Marmosa canescens (Tlacuachín) mapas consenso de los mamíferos terrestres de México. Distribución potencial. Catálogo de metadatos geográficos. Comisión Nacional para el Conocimiento y Uso de la Biodiversidad (http://www.conabio.gob.mx/informacion/gis/).

Sánchez-Cordero, V., P. Illoldi-Rangel, M. Linaje, S. Sarkar, y A. Peterson. 2005. Deforestation and extant distributions of Mexican endemic mammals. Biological Conservation 126:465-473.

Vargas-Contreras, J. A., J. R. Herrera-Herrera, y J. E. Escobedo-Cabrera, 2004. Noteworthy records of mammals from Campeche, México. Revista Mexicana de Mastozoología 8:61-69.

Velázquez, J. A., y F. J. Romero-Malpica. 1998. Análisis de la heterogeneidad ambiental y conectividad de las áreas naturales del sur del Valle de México. Universidad Nacional Autónoma de México. Facultad de Ciencias. Bases de datos SNIB2010-CONABIO proyecto No. B144. Ciudad de México, México.

Villa, R. B. y F. A. Cervantes R. 2002. Los Mamíferos de México. Editorial Iberoamérica, S. A. de C. V. Ciudad de México, México.

Zarza, H., G. Ceballos y M. A. Steele. 2003. Marmosa canescens. The American Society of Mammalogists. Mammalian Species 725:1-4.

Sometido: 26 de agosto de 2014

Revisado: 30 de septiembre de 2014

Aceptado: 8 de diciembre de 2014

Editor asociado: Rafael Reyna 